\title{
Um passeio por várias álgebras na descrição do momento angular
}

A walk through multiple algebras in the description of the moment angular

\author{
Emerson Dionísio Belançon*1], Samuel da Silva ${ }^{2}$ \\ ${ }^{1}$ Universidade Federal de Mato Grosso, Departamento de Matemática, Instituto de Ciências Exatas e Naturais, Rondonópolis, \\ MT, Brasil \\ ${ }^{2}$ Universidade Estadual Paulista, Faculdade de Engenharia de Ilha Solteira, Departamento de Engenharia Mecânica, Ilha \\ Solteira, SP, Brasil
}

Recebido em 01 de Setembro, 2018. Revisado em 28 de Setembro, 2018. Aceito em 01 de Outubro, 2018.

\begin{abstract}
A forma mais comum de definir matematicamente o momento angular é através da operação de produto vetorial como definido por Josiah Willard Gibbs e ao mesmo tempo por Oliver Heaviside. Neste sentido, a contribuição deste artigo é mostrar que a definição do momento angular também pode ser feita usando outros sistemas algébricos existentes e menos conhecidos da maioria dos estudantes, como a álgebra de Hamilton, a álgebra de Grassmann e a álgebra geométrica de Clifford. Por fim, realizamos uma comparação crítica entre estas definições e o seus significados.
\end{abstract}

Palavras-chave: momento angular, álgebra, quatérnion, vetores, multivetores, Hamilton, Grassmann, Clifford.

\begin{abstract}
The most common way to mathematically define the angular moment is through the vector product operation as defined by Josiah Willard Gibbs and at the same time by Oliver Heaviside. This way, the contribution of this article is to show that the definition of angular moment can also be made using other algebraic systems existing and less known to most students, such as Hamilton algebra, Grassmann algebra and Clifford geometric algebra. Finally, we make a critical comparison between these definitions and their meanings.
\end{abstract}

Keywords: angular moment, algebra, quaternion, vectors, multivectors, Hamilton, Grassmann, Clifford.

\section{Introdução}

O momento angular é uma grandeza associada a um objeto que executa um movimento de rotação em torno de um ponto fixo, sendo uma das principais grandezas tanto na física clássica quanto moderna, podendo ser observado em diversas situações, por exemplo, Lekner 1] mostra que os pulsos sonoros transportam momento angular e são conservados quando o amortecimento e o espalhamento viscosos são desprezados. Nessa mesma linha Wunenburger, Lozano e Brasselet 2 realizaram uma demonstração experimental da troca de momento angular orbital entre som e matéria medida por um processo de espalhamento quiral não dissipativo. Na mecânica quântica, Singh e Mobed [3] afirmam que a curvatura do espaço-tempo local pode contribuir de forma não trivial para as propriedades do momento angular orbital já em [4] há uma discussão de novos resultados sobre a conversão spin-orbital do momento angular orbital da luz e em [5], um estudo experimental mostra a reconstrução do spin e do momento angular orbital da luz após distorção em amplitude, fase e estado de polarização.

Apesar do momento angular ter uma expressão aparentemente simples muitos estudantes encontram difi-

*Endereço de correspondência: edbelancon@ufmt.br culdades quando iniciam o estudo de rotação de sólidos. Essas dificuldades estão relacionadas diretamente com o conceito de momento angular e a velocidade angular. Uma das causas destas dificuldades são conceituais e deve-se ao fato de que as leis que governam a translação e rotação são semelhantes [6,7]. Por outro lado, vários experimentos podem ser feitos para que os alunos possam visualizar e demonstrar o momento angular $8-10$ ou a conservação do momento angular [11 14]. Aliado a isto, a própria descrição matemática do momento angular, dependendo do sistema álgebrico usado pode dificultar ou facilitar o entendimento dos signficados e a precisão deste conceito aos estudantes.

Neste sentido, o presente artigo apresenta uma descrição matemática do conceito de momento angular usando diferentes sistemas algébricos. O objetivo aqui é definilo usando quatro álgebras diferentes: a álgebra vetorial, com base em Gibbs-Heaviside, a álgebra de Hamilton, a álgebra de Grassmann e a álgebra geométrica de Clifford, apresentando uma comparação entre estas definições que não são comumente abordados em livros de física. Não serão abordados as interfaces entre estas álgebras, pois esse assunto já foi, e continua sendo, bastante discutido na literatura como em 15 19]. 
Na primeira seção é feito uma sucinta revisão dos principais conceitos para definir a álgebra vetorial de Gibbs-Heaviside. Logo após, é colocado uma descrição detalhada do momento angular frisando os conceitos que serão usados para uma melhor compreensão da sua descrição com as outras álgebras partindo da criação histórica de cada uma delas e como se interpreta o conceito de produto entre vetores. No final, apresenta-se uma comparação crítica do momento angular escrito nestas quatro álgebras apontando a melhor forma para sua representação matemática e uso prático em problemas da física.

\section{A Álgebra Vetorial}

Por volta de 1883, Josiah Willard Gibbs fez uma análise da Álgebra de Grassmann assim como a álgebra de Hamilton [20] e pôde simplificá-las de forma considerável. Gibbs apresentou seus estudos sob a forma de notas de aula que utilizava junto aos seus alunos 21. que posteriormente foi publicada em forma de livro 22 . Ao mesmo tempo, Oliver Heaviside, chegou a resultados semelhantes ao de Gibbs, de forma independente, mas nunca escreveu um tratado formal sobre análise vetorial. Os resultados desenvolvidos sobre vetores foram introduzidos em seus artigos sobre eletromagnetismo que depois foram reunidos em dois volumes chamados Electrical $P a$ pers 23]. Estes acontecimentos marcam o nascimento da chamada álgebra vetorial conhecida também como álgebra de Gibbs-Heaviside.

Nesta álgebra os elementos existentes são escalares e os vetores. Os vetores se dividem em duas classes: os vetores polares, ou simplesmente vetores, que possuem direção e sentido natural e os vetores axiais ou pseudovetores ${ }^{1}$ que possuem direção e sentido convencionados pela regra da mão direita (ou regra de Fleming $\left.{ }^{2}\right]$ [24] na operação de produto vetorial entre eles. A definição da álgebra vetorial pode ser encontrada em uma vasta lista de livros básicos de geometria 25,26].

Para definir os elementos desta álgebra toma-se o espaço euclidiano tridimensional $\mathbb{R}^{3}$ e uma base ortonormal $\mathcal{B}=\left\{\hat{e}_{1}, \hat{e}_{2}, \hat{e}_{3}\right\}$ neste espaço. Assim, a representação de um vetor $\mathbf{w} \in \mathbb{R}^{3}$ é dada simplesmente por $\mathbf{w}=x \hat{e}_{1}+y \hat{e}_{2}+z \hat{e}_{3} \operatorname{com} x, y, z \in \mathbb{R}$. O módulo de um vetor é dado por

$$
|\mathbf{w}|=\sqrt{x^{2}+y^{2}+z^{2}} .
$$

Gibbs, ao mesmo tempo que Heaviside, definiram dois produtos entre estes elementos. Sejam $\mathbf{w}, \mathbf{u} \in \mathbb{R}^{3}$ e $\alpha$ o menor ângulo entre eles. Para $\alpha \neq 0$, w e u definem um plano e o produto escalar entre eles, representado por $\mathbf{w} \cdot \mathbf{u}$, é um número real definido por

$$
\mathbf{w} \cdot \mathbf{u}=|\mathbf{w} \| \mathbf{u}| \cos \alpha .
$$

${ }^{1}$ Os adjetivos polares e axiais foram introduzidos por Woldemar Voigt em 1896.

${ }^{2}$ Criada originalmente pelo físico John Ambrose Fleming
A interpretação geométrica desse produto pode ser vista como a multiplicação do módulo de w com módulo da componente de $\mathbf{u}$ que tem mesma direção de $\mathbf{w}$ como ilustrado na figura (1). Esse produto está relacionado com comprimento uma vez que ele amplia/reduz o comprimento de um dos vetores. Segue que o produto escalar é comutativo mas não associativo e satisfaz as seguintes relações entre os vetores da base $\mathcal{B}$

$$
\begin{array}{lll}
\hat{e}_{i} \cdot \hat{e}_{i}=1 & \text { para } & i=1,2,3 \\
\hat{e}_{i} \cdot \hat{e}_{j}=\hat{e}_{j} \cdot \hat{e}_{i}=0 & \text { para } & i \neq j .
\end{array}
$$

Já o produto vetorial entre esses vetores, representado por $\mathbf{w} \times \mathbf{u}$, é um vetor definido por

$$
\mathbf{w} \times \mathbf{u}=|\mathbf{w} \| \mathbf{u}| \sin \alpha \hat{n},
$$

onde $\hat{n}$ é um vetor unitário cujo sentido é normal ao plano formado por w e $\mathbf{u}$ e direção satisfazendo a regra da mão direita e com módulo dado por

$$
|\mathbf{w} \times \mathbf{u}|=|\mathbf{w}||\mathbf{u}| \sin \alpha .
$$

A interpretação geométrica do módulo desse produto pode ser vista como a multiplicação do módulo de w com módulo da componente de u que é perpendicular a direção de w, também ilustrado na figura (1). Esse produto está relacionado com o valor da área do paralelogramo formado por esses vetores já que a componente perpendicular é a altura deste paralelogramo.

Segue que o produto vetorial, por sua vez, não é comutativo e nem associativo e satisfaz as seguintes relações entre os vetores da base $\mathcal{B}$

$$
\begin{array}{lll}
\hat{e}_{i} \times \hat{e}_{i}=0 & \text { para } & i=1,2 \text { ou } 3 \\
\hat{e}_{i} \times \hat{e}_{j}=\varepsilon_{i j k} \hat{e}_{k} & \text { para } & i \neq j \neq k
\end{array}
$$

sendo $\varepsilon_{i j k}=1$ se $i j k=123,231$ ou 312 (permutação cíclica) ou $\varepsilon_{i j k}=-1$ se $i j k=213,132$ ou 321 (permutação acíclica) o que dá origem a conhecida regra mnemônica da mão direita.

Com isso, dado os vetores $\mathbf{w}=x_{1} \hat{e}_{1}+x_{2} \hat{e}_{2}+x_{3} \hat{e}_{3}$ e $\mathbf{u}=y_{1} \hat{e}_{1}+y_{2} \hat{e}_{2}+y_{3} \hat{e}_{3}$ segue das relações (1), (3) e das propriedades associativa e distributiva dos números reais as expressões conhecidas para os produtos escalar e vetorial, respectivamente

$$
\mathbf{w} \cdot \mathbf{u}=x_{1} y_{1}+x_{2} y_{2}+x_{3} y_{3} .
$$

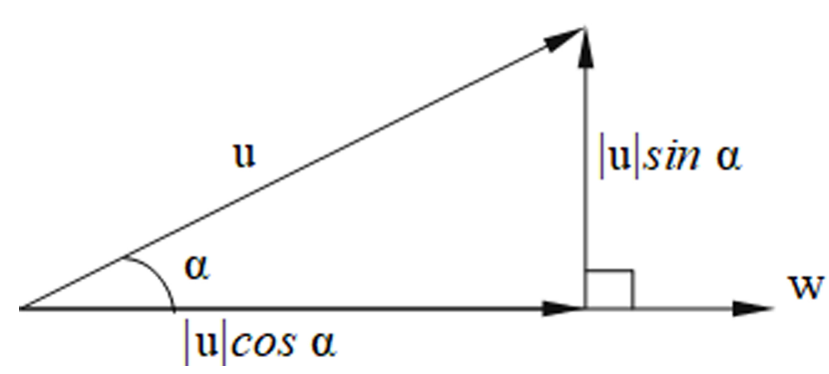

Figura 1: Interpretação geométrica do produto escalar e do módulo do produto vetorial. 


$$
\begin{aligned}
\mathbf{w} \times \mathbf{u} & =\left(x_{2} y_{3}-x_{3} y_{2}\right) \hat{e}_{1}+\left(x_{3} y_{1}-x_{1} y_{3}\right) \hat{e}_{2} \\
& +\left(x_{1} y_{2}-x_{2} y_{1}\right) \hat{e}_{3} .
\end{aligned}
$$

Em qualquer livro de física, por exemplo [27 28], podese encontrar a definição do momento angular de uma partícula usando o conceito anterior de produto vetorial. Com esta álgebra o momento angular de uma partícula de massa $m$, denotado por $\mathscr{H}$, com relação ao ponto $O$ movendo-se com velocidade $\mathbf{v}$ é calculado como

$$
\mathscr{H}=\mathbf{r} \times \mathbf{p}=m(\mathbf{r} \times \mathbf{v})
$$

sendo $\mathbf{r}$ o vetor com origem no ponto $O$ até a posição da partícula e $\mathbf{p}=m \mathbf{v}$ é o momento linear desta partícula. Decorre que o momento angular é normal ao plano formado pelos vetores $\mathbf{r}$ e p. Geometricamente, o momento angular pode ser representado como o expresso na figura (2).

Se um vetor posição é $\mathbf{r}=r_{1} \hat{e}_{1}+r_{2} \hat{e}_{2}+r_{3} \hat{e}_{3}$ e um vetor velocidade é $\mathbf{v}=v_{1} \hat{e}_{1}+v_{2} \hat{e}_{2}+v_{3} \hat{e}_{3}$, segue da equação (5) que a representação algébrica do momento angular será

$$
\begin{aligned}
\mathscr{H} & =m\left[\left(r_{2} v_{3}-r_{3} v_{2}\right) \hat{e}_{1}+\left(r_{3} v_{1}-r_{1} v_{3}\right) \hat{e}_{2}\right. \\
& \left.+\left(r_{1} v_{2}-r_{2} v_{1}\right) \hat{e}_{3}\right]
\end{aligned}
$$

que é a forma padrão com que a totalidade dos estudantes tem contato com a definição do momento angular. Porém, como abordamos neste artigo, outras formas menos conhecidas do grande público podem ser usadas para descrever o mesmo conceito físico do momento angular.

\section{A Álgebra de Hamilton}

Bem antes dos trabalhos de Gibbs e Heaviside, William Rowan Hamilton construiu no século XIX um novo conjunto de objetos com propriedades semelhantes aos números complexos tentando generaliza-los para o espaço tridimensional com uma estrutura fechada. Esses novos objetos com quatro componentes sendo uma escalar e

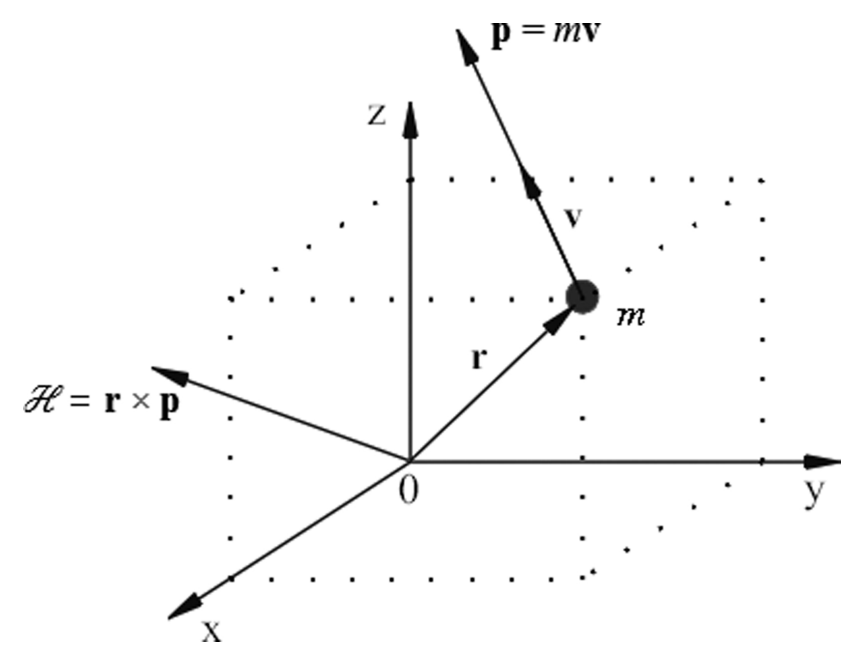

Figura 2: Representação geométrica dos momentos linear e angular três unidades imaginárias foi por ele denominado quatérnions e publicado no livro Elements of quaternions 29. em 1843. Com este formalismo, as regras válidas para os números complexos eram satisfeitas, com a única diferença de que o produto entre quatérnions não é uma operação comutativa.

O conjunto dos quatérnions, denotado por $\mathbb{H}$, satisfaz as propriedades de espaço vetorial sobre o corpo dos números reais. Um quatérnion pode ser representado, conforme Kuipers 30], por

$$
\widetilde{q}=q_{0}+q_{1} \mathbf{i}+q_{2} \mathbf{j}+q_{3} \mathbf{k},
$$

sendo $q_{0}, q_{1}, q_{2}$ e $q_{3} \in \mathbb{R}$ os componentes de $\widetilde{q}$ e $\mathbf{i}, \mathbf{j}$ e $\mathbf{k}$ as unidades imaginárias que satisfazem as seguintes relações fundamentais

$$
\begin{aligned}
& \mathbf{i}^{2}=\mathbf{j}^{2}=\mathbf{k}^{2}=-1 \\
& \mathbf{i j}=\mathbf{k}=-\mathbf{j i} \\
& \mathbf{j} \mathbf{k}=\mathbf{i}=-\mathbf{k j} \\
& \mathbf{k i}=\mathbf{j}=-\mathbf{i k} .
\end{aligned}
$$

Um quatérnion $\widetilde{q}$ possui uma parte real, chamada por Hamilton de parte escalar, denotada por $S(\widetilde{q})=q_{0}$ e uma parte imaginária, conhecida como parte vetorial ou simplesmente vetor, denotada por $\mathbf{V}(\widetilde{q})=q_{1} \mathbf{i}+q_{2} \mathbf{j}+q_{3} \mathbf{k}$. Assim, um quatérnion pode ser escrito simplesmente como $\widetilde{q}=S(\widetilde{q})+\mathbf{V}(\widetilde{q})$. Se $\mathbf{V}(\widetilde{q})=0$, então $\widetilde{q}=S(\widetilde{q})$ é um número real; agora se $S(\widetilde{q})=0$, então $\widetilde{q}=\mathbf{V}(\widetilde{q})$ é dito um quatérnion puro (vetor). Hamilton também definiu o produto entre quatérnions utilizando as regras dos números complexos e alguns relações fundamentais, descritas pelas equações (8) envolvendo as unidades imaginárias. Aqui será considerado o caso particular de quatérnions puros que se mostram suficientes para a definição do momento angular. Dados os quatérnions $\widetilde{q}=0+\mathbf{w}=x_{1} \mathbf{i}+x_{2} \mathbf{j}+x_{3} \mathbf{k}$ e $\widetilde{p}=0+\mathbf{u}=y_{1} \mathbf{i}+y_{2} \mathbf{j}+y_{3} \mathbf{k}$, o produto entre eles é dado por

$$
\begin{aligned}
\widetilde{q} \widetilde{p}=\mathbf{w u} & =-\left(x_{1} y_{1}+x_{2} y_{2}+x_{3} y_{3}\right) \\
& +\left(x_{2} y_{3}-x_{3} y_{2}\right) \mathbf{i}+\left(x_{3} y_{1}-x_{1} y_{3}\right) \mathbf{j} \\
& +\left(x_{1} y_{2}-x_{2} y_{1}\right) \mathbf{k}
\end{aligned}
$$

O espaço vetorial real $\mathbb{H}$ equipado com o produto entre quatérnions é dito uma álgebra, denominada álgebra de Hamilton. A álgebra de Hamilton é associativa em relação ao produto mas não é comutativa que claramente pode ser visto nas relações (8), porém é uma álgebra fechada. Como o objetivo é escrever o momento angular será considerado o vetor posição $\mathbf{r}$ e o vetor velocidade $\mathbf{v}$ como quatérnions puros da forma $\widetilde{r}=0+\mathbf{r}=r_{1} \mathbf{i}+r_{2} \mathbf{j}+r_{3} \mathbf{k}$ e $\widetilde{v}=0+\mathbf{v}=v_{1} \mathbf{i}+v_{2} \mathbf{j}+v_{3} \mathbf{k}$.

Vale observar que foi utilizado na álgebra de Hamilton as mesmas componentes dos vetores $\mathbf{r}$ e $\mathbf{v}$ da álgebra vetorial. Isto significa considerar uma equivalência entre os vetores da base $\mathcal{B}$ e as unidades imaginárias $\mathbf{i}, \mathbf{j}$ e $\mathbf{k}$. Apesar de Hamilton ter chamado estas unidades de "versor perpendicular"e defini-los a partir de um conjunto de três vetores unitários perpendiculares, Hamilton passou a usá-los como conjuntos equivalentes mesmo tendo 
significado diferente. Assim, o produto entre quatérnions puro é dado pela expressão

$$
\begin{aligned}
\tilde{r} \widetilde{v}=\mathbf{r v} & =-\left(r_{1} v_{1}+r_{2} v_{2}+r_{3} v_{3}\right) \\
& +\left(r_{2} v_{3}-r_{3} v_{2}\right) \mathbf{i}+\left(r_{3} v_{1}-r_{1} v_{3}\right) \mathbf{j} \\
& +\left(r_{1} v_{2}-r_{2} v_{1}\right) \mathbf{k}
\end{aligned}
$$

O produto de dois quatérnions puros tem como resultado a estrutura de um quatérnion geral (parte escalar e vetorial). A interpretação geométrica desse produto via rotações no espaço só foi feito por Hamilton em 1844 com o artigo [31]. Inicialmente Hamilton decompôs $\mathbf{v}$ nos vetores $\mathbf{v}_{\|}$e $\mathbf{v}_{\perp}$ onde $\mathbf{v}_{\|}$é a projeção de $\mathbf{v}$ na direção de $\mathbf{r}$ e $\mathbf{v}_{\perp}$ é a projeção de $\mathbf{v}$ em uma direção perpendicular à $\mathbf{r}$, como ilustrado na figura (3).

Admitindo que a multiplicação entre vetores é distributiva, Hamilton obteve

$$
\widetilde{r} \widetilde{v}=\mathbf{r v}=\mathbf{r}\left(\mathbf{v}_{\|}+\mathbf{v}_{\perp}\right)=\mathbf{r} \mathbf{v}_{\|}+\mathbf{r} \mathbf{v}_{\perp}
$$

e definiu os produtos parciais $\mathbf{r} \mathbf{v}_{\|}$e $\mathbf{r v}_{\perp}$ da seguinte maneira:

- $\mathbf{r} \mathbf{v}_{\|}$é um escalar dado pelo produto dos comprimentos dos vetores $\mathbf{r}$ e $\mathbf{v}_{\|}$, antecedido por um sinal negativo se estes possuem o mesmo sentido ou por sinal positivo, se possuem sentido contrário.

- $\mathbf{r v}_{\perp}$ é um vetor cujo comprimento é o produto dos comprimentos de $\mathbf{r}$ e $\mathbf{v}_{\perp}$ cuja direção é perpendicular a ambos.

Pode-se observar na equação 10 que a parte vetorial tem a mesma estrutura que a equação (7), além disso, de acordo com a interpretação geométrica de Hamilton, a parte vetorial deste resultado descreve a mesma grandeza da equação (7) já que possuem mesmo módulo, direção e sentido. Portanto, o momento angular pode ser considerado como a parte vetorial do produto entre os dois quatérnions $\widetilde{r}$ e $\widetilde{v}$, isto é, $\mathscr{H}=m[\mathbf{V}(\widetilde{r} \widetilde{v})]$.

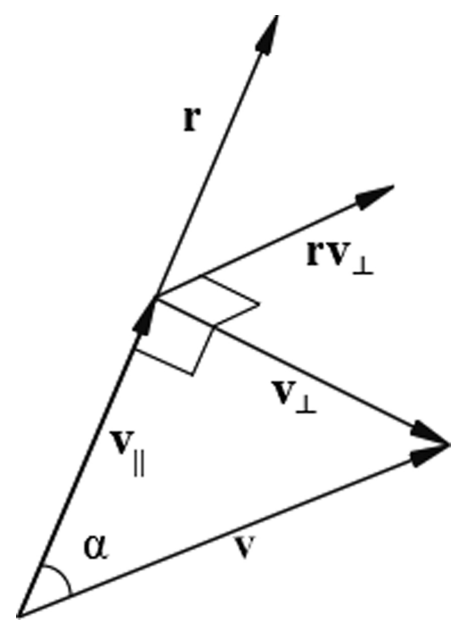

Figura 3: Representação geométrica de Hamilton.

\section{A Álgebra de Grassmann}

Com a descoberta de Hamilton outros pesquisadores se interessaram em pesquisar novos objetos matemáticos, entre eles, Hermann Günther Grassmann teve importância fundamental. O primeiro volume do principal trabalho de Grassmann foi apresentado em 1843, na forma manuscrita, com o nome de Die Lineale Ausdehnungslehr $\mathrm{t}^{3}$ e publicada em 1844 32. Neste trabralho, Grassmann propôs representar as grandezas físicas por objetos geométricos ao invés de números. Estes objetos seriam como retas orientadas, fragmentos de plano orientados, cubos orientados, etc. Pode-se dizer que foi exatamente neste ponto que surgiu o conceito de objetos vetoriais, como é conhecido hoje. O trabalho de Grassmann foi, sem dúvida, excepcional, entretanto, o seu trabalho era por demais complexo para a época em que foi publicado, e poucos foram os que o entenderam e deram importância na época.

A álgebra de Grassmann pode ser construída a partir de aplicações lineares alternadas ou a partir do quociente de alguma álgebra tensorial. Porém, nosso objetivo é saber como calcular o produto entre vetores para descrever o momento angular. Sendo assim, será colocado apenas as principais definições sem entrar em conceitos mais gerais. O leitor interessado pode consultar as referências 19,33 para detalhes aprofundados.

Dado um tensor na forma $\mathbf{v}_{1} \otimes \mathbf{v}_{2} \otimes \ldots \otimes \mathbf{v}_{p}$, em que $\mathbf{v}_{i}$ com $i=1, \ldots, p$ são vetores de um espaço vetorial $V$ sobre o corpo dos reais $\mathbb{R}$ e $\otimes$ denota o produto tensorial, pode-se definir o operador alternador, denotado por Alt, como segue

$$
\begin{aligned}
& \operatorname{Alt}\left(\mathbf{v}_{1} \otimes \mathbf{v}_{2} \otimes \ldots \otimes \mathbf{v}_{p}\right)= \\
& \frac{1}{p !} \sum_{\sigma \in S_{p}} \varepsilon(\sigma) \mathbf{v}_{\sigma(1)} \otimes \mathbf{v}_{\sigma(2)} \otimes \ldots \otimes \mathbf{v}_{\sigma(p)},
\end{aligned}
$$

onde $S_{p}$ é o conjunto de todas as permutações possíveis e $\varepsilon(\sigma)=1$, se o número de permutações feitas for par, e $\varepsilon(\sigma)=-1$, se o número de permutações feitas for ímpar. Nestas condições, um p-vetores, denotado por $A_{p}$, é um tensor contravariante 4 de ordem $p$ (ou grade $p$ ) alternado

$$
A_{p}=\operatorname{Alt}\left(\mathbf{v}_{1} \otimes \mathbf{v}_{2} \otimes \ldots \otimes \mathbf{v}_{p}\right) \text {, }
$$

onde $\mathbf{v}_{1}, \mathbf{v}_{2}, \ldots, \mathbf{v}_{p} \in V$.

Denota-se por $\bigwedge^{p}(V)$ o conjunto dos p-vetores. Tal conjunto possui a estrutura de espaço vetorial. É possível definir um produto entre elementos desses espaços. Sejam $A_{p} \in \bigwedge^{p}(V)$ um p-vetor e $B_{q} \in \bigwedge^{q}(V)$ um q-vetor. O produto exterior é dado por

$$
\begin{aligned}
& \wedge: \bigwedge^{p}(V) \times \bigwedge^{q}(V) \longrightarrow \bigwedge^{p+q}(V) \\
& A_{p} \wedge B_{q} \quad \longmapsto \quad \operatorname{Alt}\left(A_{p} \otimes B_{q}\right),
\end{aligned}
$$

\footnotetext{
${ }^{3}$ A Teoria das Extensões Lineares

${ }^{4}$ São tensores do tipo $(\mathrm{p}, \mathrm{q})$ - por exemplo um vetor é um tensor contravariante do tipo $(0,1)$.
} 
este produto também é conhecido como cunha ou de Grassmann e é associativo.

Das equações 12 e (14) pode-se calcular o produto exterior de dois vetores $\mathbf{w}$ e $\mathbf{u}$

$$
\mathbf{w} \wedge \mathbf{u}=\frac{1}{2}(\mathbf{w} \otimes \mathbf{u}-\mathbf{u} \otimes \mathbf{w})
$$

claramente é possível concluir que o produto exterior é não comutativo.

Assumindo o espaço euclidiano tridimensional $V=\mathbb{R}^{3}$ e uma base ortonormal $\mathcal{B}=\left\{\hat{e}_{1}, \hat{e}_{2}, \hat{e}_{3}\right\}$, conclui-se que os vetores de $\mathcal{B}$ satisfazem as seguintes relações

$$
\begin{array}{lll}
\hat{e}_{i} \wedge \hat{e}_{i}=0 & \text { para } & i=1,2,3 \\
\hat{e}_{i} \wedge \hat{e}_{j}=-\hat{e}_{j} \wedge \hat{e}_{i} & \text { para } & i \neq j .
\end{array}
$$

Além disso, pode-se construir quatro espaços vetoriais a partir $\mathbb{R}^{3}$, isto é, para $p=0, \ldots, 3$ têm-se $\bigwedge^{0}\left(\mathbb{R}^{3}\right)=\mathbb{R}$ espaço dos escalares, $\Lambda^{1}\left(\mathbb{R}^{3}\right)=\mathbb{R}^{3}$ espaço dos 1 -vetores (ou vetores), $\bigwedge^{2}\left(\mathbb{R}^{3}\right)$ espaço dos 2-vetores (ou bivetores), $\bigwedge^{3}\left(\mathbb{R}^{3}\right)$ espaço dos 3-vetores (ou trivetores).

Os escalares e vetores são os velhos conhecidos da álgebra vetorial, mas os bivetor e trivetor são os objetos que diferenciam a álgebra de Grassmann uma vez que estes estão relacionados com área e volume que não encontram correspondentes na álgebra de Gibbs-Heaviside. Uma base para o espaço dos bivetores é dado por todas as permutações da forma $\hat{e}_{i} \wedge \hat{e}_{j}$ dos elementos de $\mathcal{B}$ que são linearmente independentes. Um conjunto dessas permutações é dado por $\left\{\hat{e}_{1} \wedge \hat{e}_{2}, \hat{e}_{1} \wedge \hat{e}_{3}, \hat{e}_{2} \wedge \hat{e}_{3}\right\}$. Assim, um bivetor pode ser escrito, algebricamente, na forma

$$
A_{2}=a_{12} \hat{e}_{1} \wedge \hat{e}_{2}+a_{13} \hat{e}_{1} \wedge \hat{e}_{3}+a_{23} \hat{e}_{2} \wedge \hat{e}_{3}
$$

e, geometricamente, pode ser representado como um fragmento de plano orientado gerado por dois vetores ordenados, conforme a figura (4).

Já uma base para o espaço dos trivetores é qualquer permutação da forma $\hat{e}_{i} \wedge \hat{e}_{j} \wedge \hat{e}_{k}$. Assim, um trivetor
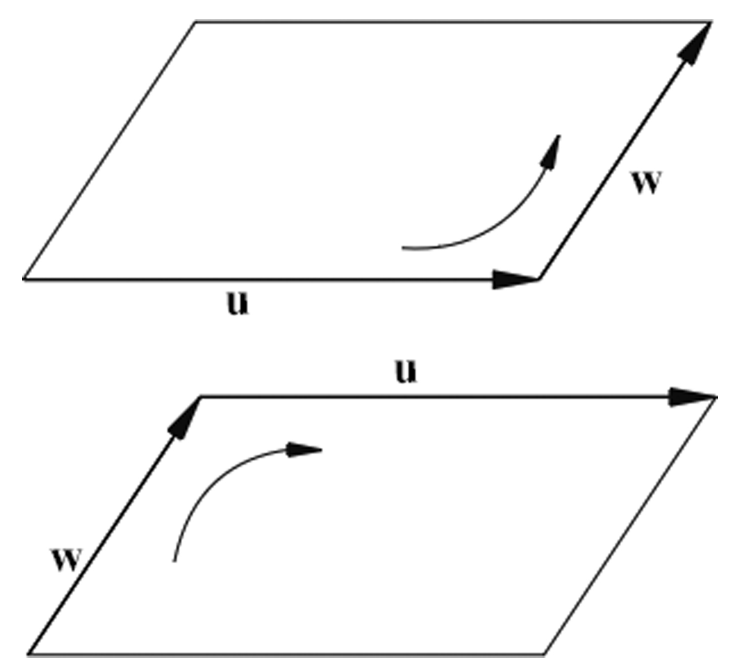

Figura 4: Representação geométrica das duas orientações possíveis para um bivetor. pode ser escrito, algebricamente, na forma

$$
A_{3}=a_{123} \hat{e}_{1} \wedge \hat{e}_{2} \wedge \hat{e}_{3}
$$

e, geometricamente, pode ser representado como um volume orientado, conforme a figura (5).

Ao aplicar o produto exterior em um p-vetor e um qvetor, equação (14), o resultado é um $p+q$-vetor que não pertence ao espaço $\bigwedge^{p}(V)$ e nem ao espaço $\bigwedge^{q}(V)$. Para resolver o problema do não fechamento deste produto, pode-se considerar a soma direta dos quatros espaços vetoriais construídos a partir de $\mathbb{R}^{3}$, isto é,

$$
\bigwedge\left(\mathbb{R}^{3}\right)=\bigoplus_{p=0}^{3} \bigwedge^{p}\left(\mathbb{R}^{3}\right)
$$

no qual é fechado em relação ao produto exterior. Este espaço é denominado espaço multivetorial e cada elemento arbitrário $A \in \bigwedge\left(\mathbb{R}^{3}\right)$ é chamado de multivetor e pode ser escrito de maneira geral como

$$
\begin{aligned}
A & =a_{0}+a_{1} \hat{e}_{1}+a_{2} \hat{e}_{2}+a_{3} \hat{e}_{3}+a_{12} \hat{e}_{1} \wedge \hat{e}_{2} \\
& =+a_{13} \hat{e}_{1} \wedge \hat{e}_{3}+a_{23} \hat{e}_{2} \wedge \hat{e}_{3}+a_{123} \hat{e}_{1} \wedge \hat{e}_{2} \wedge \hat{e}_{3} .
\end{aligned}
$$

Tal espaço dotado do produto exterior é denominado álgebra exterior do espaço vetorial $\mathbb{R}^{3}$. Em muitos textos é considerado a álgebra exterior e a álgebra de Grassmann como sinônimos, porém existe uma distinção entre as duas estruturas, a álgebra de Grassmann é a álgebra exterior equipado com uma generalização de um funcional linear $g: V \times V \rightarrow \mathbb{R}$ para o espaço vetorial $\bigwedge(V)$. Como o interesse é no produto entre dois vetores, o produto exterior é calculado de forma análoga na álgebra de Grassmann e não vamos adentrar no rigor das definições, que podem ser encontradas em lingua portuguesa em [19].

O módulo de um bivetor pode ser dado como a área do paralelogramo formado pelo produto exterior entre dois vetores, isto é

$$
|\mathbf{u} \wedge \mathbf{w}|=|\mathbf{u}||\mathbf{w}| \sin \alpha
$$

com a interpretação geométrica descrita na figura (6).

Pode-se definir um produto métrico, denominado contração à esquerda.$^{5}$ entre $p$-vetores equivalente ao produto escalar quando restrito à vetores.

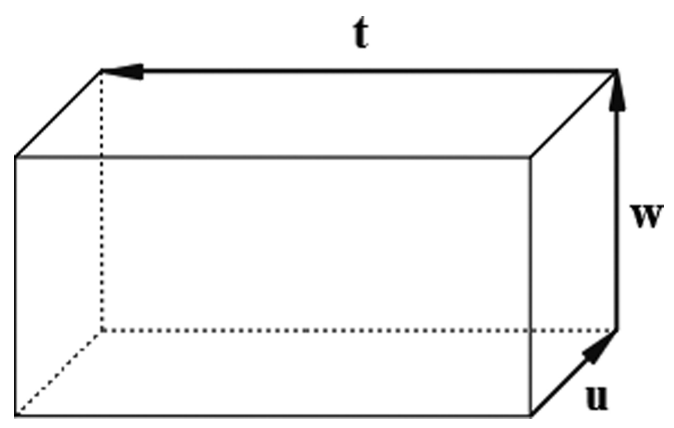

Figura 5: Representação geométrica do trivetor $\mathbf{u} \wedge \mathbf{w} \wedge \mathbf{t}$.

${ }^{5}$ Pode-se definir a contração à direita de forma anóloga e denotada por $\lfloor$. A contração também é muitas vezes denominada de produto interno quando calculada entre vetores. 


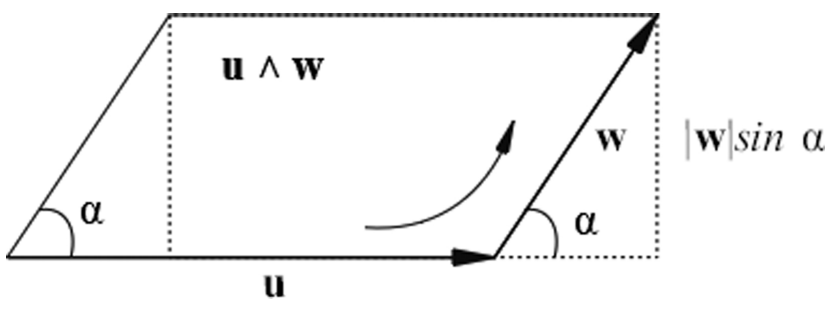

Figura 6: Representação geométrica do módulo de um bivetor.

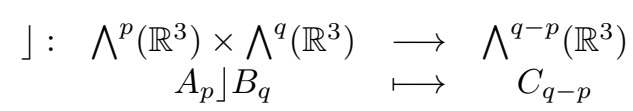

Este produto não é associativo e só é simétrico se $p=q$ porém satisfaz as seguintes propriedades:

i) $\alpha\rfloor A_{p}=\alpha A_{p}$;

ii) $\mathbf{u}\rfloor \mathbf{w}=\mathbf{u} \cdot \mathbf{w}$

iii) $\left.\left.\mathbf{u}\rfloor\left(B_{q} \wedge C_{k}\right)=(\mathbf{u}\rfloor B_{q}\right) \wedge C_{k}+(-1)^{q} B_{q} \wedge(\mathbf{u}\rfloor C_{k}\right)$;

iv) $\left.\left.\left.\left(A_{p} \wedge B_{q}\right)\right\rfloor C_{k}=A_{p}\right\rfloor\left(B_{q}\right\rfloor C_{k}\right)$.

onde $\alpha$ é um escalar, u e w são vetores e $A_{p}, B_{q}, C_{k}$ um $p$-vetor um $q$-vetor e um $k$-vetor respectivamente.

Com isto, defini-se uma operação denominada dualidade ou como em [19] isomorfismo quase-Hodge como segue

$$
\begin{aligned}
& \star: \bigwedge^{p}\left(\mathbb{R}^{3}\right) \longrightarrow \bigwedge^{3-p}\left(\mathbb{R}^{3}\right) \\
& \left.\star A_{p} \quad \longmapsto \quad-A_{p}\right] \mathbb{I}_{3}
\end{aligned}
$$

onde $\mathbb{I}_{3}$ representa um trivetor simples unitário, isto é, $\mathbb{I}_{3}=\hat{e}_{1} \wedge \hat{e}_{2} \wedge \hat{e}_{3}$ denominado como pseudoescalar.

Por exemplo, para calcular o dual do bivetor $A_{2}=$ $\hat{e}_{1} \wedge \hat{e}_{2}$ deve ser aplicado, além da definição (18), as propriedades da contração, ou seja,

$$
\begin{aligned}
\star A_{2}= & \left.-\left(\hat{e}_{1} \wedge \hat{e}_{2}\right)\right\rfloor\left(\hat{e}_{1} \wedge \hat{e}_{2} \wedge \hat{e}_{3}\right) \\
= & \left.\left.-\left\{\hat{e}_{1}\right\rfloor\left(\hat{e}_{2}\right\rfloor\left(\hat{e}_{1} \wedge\left(\hat{e}_{2} \wedge \hat{e}_{3}\right)\right)\right)\right\} \\
= & \left.-\left\{\hat{e}_{1}\right\rfloor\left(\hat{e}_{2}\right\rfloor \hat{e}_{1}\right) \wedge\left(\hat{e}_{2} \wedge \hat{e}_{3}\right) \\
& \left.\left.-\hat{e}_{1} \wedge\left(\hat{e}_{2}\right\rfloor\left(\hat{e}_{2} \wedge \hat{e}_{3}\right)\right)\right\} \\
= & \left.-\left\{\hat{e}_{1}\right\rfloor\left(\hat{e}_{2}\right\rfloor \hat{e}_{1}\right) \wedge\left(\hat{e}_{2} \wedge \hat{e}_{3}\right) \\
& \left.\left.\left.-\hat{e}_{1} \wedge\left[\left(\hat{e}_{2}\right\rfloor \hat{e}_{2}\right) \wedge \hat{e}_{3}-\hat{e}_{3} \wedge\left(\hat{e}_{2}\right\rfloor \hat{e}_{1}\right)\right]\right\} \\
= & \left.-\left\{\hat{e}_{1}\right\rfloor\left(-\hat{e}_{1} \wedge \hat{e}_{3}\right)\right\} \\
= & \left.\left.-\left\{-\hat{e}_{1}\right\rfloor \hat{e}_{1} \wedge \hat{e}_{3}+\hat{e}_{1} \wedge\left(\hat{e}_{1}\right\rfloor \hat{e}_{3}\right)\right\} \\
= & -\left\{-\hat{e}_{3}\right\}=\hat{e}_{3}
\end{aligned}
$$

Com isso, a representação dos vetores posição $\mathbf{r}$ e velocidade $\mathbf{v}$ no espaço multivetorial $\bigwedge\left(\mathbb{R}^{3}\right)$ pode ser escritos da seguinte forma $\mathbf{r}=r_{1} \hat{e}_{1}+r_{2} \hat{e}_{2}+r_{3} \hat{e}_{3}$ e $\mathbf{v}=$ $v_{1} \hat{e}_{1}+v_{2} \hat{e}_{2}+v_{3} \hat{e}_{3}$. Segue do produto exterior entre vetores, (15), que

$$
\mathbf{r} \wedge \mathbf{v}=\frac{1}{2}(\mathbf{r} \otimes \mathbf{v}-\mathbf{v} \otimes \mathbf{r})
$$

Desenvolvendo os termos

$$
\begin{aligned}
\mathbf{r} \otimes \mathbf{v} & =r_{1} v_{1} \hat{e}_{1} \wedge \hat{e}_{1}+r_{1} v_{2} \hat{e}_{1} \wedge \hat{e}_{2}+r_{1} v_{3} \hat{e}_{1} \wedge \hat{e}_{3} \\
& +r_{2} v_{1} \hat{e}_{2} \wedge \hat{e}_{1}+r_{2} v_{2} \hat{e}_{2} \wedge \hat{e}_{2}+r_{2} v_{3} \hat{e}_{2} \wedge \hat{e}_{3} \\
& +r_{3} v_{1} \hat{e}_{3} \wedge \hat{e}_{1}+r_{3} v_{2} \hat{e}_{3} \wedge \hat{e}_{2}+r_{3} v_{3} \hat{e}_{3} \wedge \hat{e}_{3} \\
\mathbf{v} \otimes \mathbf{r} & =v_{1} r_{1} \hat{e}_{1} \wedge \hat{e}_{1}+v_{1} r_{2} \hat{e}_{1} \wedge \hat{e}_{2}+v_{1} r_{3} \hat{e}_{1} \wedge \hat{e}_{3} \\
& +v_{2} r_{1} \hat{e}_{2} \wedge \hat{e}_{1}+v_{2} r_{2} \hat{e}_{2} \wedge \hat{e}_{2}+v_{2} r_{3} \hat{e}_{2} \wedge \hat{e}_{3} \\
& +v_{3} r_{1} \hat{e}_{3} \wedge \hat{e}_{1}+v_{3} r_{2} \hat{e}_{3} \wedge \hat{e}_{2}+v_{3} r_{3} \hat{e}_{3} \wedge \hat{e}_{3}
\end{aligned}
$$

e aplicando as relações (16) nestes termos e substituindo o resultado em 19 tem-se finalmente

$$
\begin{aligned}
\mathbf{r} \wedge \mathbf{v} & =\left(r_{1} v_{2}-r_{2} v_{1}\right) \hat{e}_{1} \wedge \hat{e}_{2}+\left(r_{1} v_{3}-r_{3} v_{1}\right) \hat{e}_{1} \wedge \hat{e}_{3} \\
& +\left(r_{2} v_{3}-r_{3} v_{2}\right) \hat{e}_{2} \wedge \hat{e}_{3}
\end{aligned}
$$

Mesmo sabendo que o produto entre vetores na álgebra de Grassmann resulta em um bivetor que por sua vez está relacionado com área assim como o momento angular isto não é suficiente para dizer que ambos representam a mesma grandeza. Além disto, a comparação das equações (20) e (7) não podem ser feita pois possuem grades diferentes. Mas com a operação de dualidade é possível encontrar um representante do bivetor $\mathbf{r} \wedge \mathbf{v}$ no espaço dos vetores para tal comparação. Desta forma,

$$
\begin{aligned}
\star(\mathbf{r} \wedge \mathbf{v}) & =\left(r_{2} v_{3}-r_{3} v_{2}\right) \hat{e}_{1}+\left(r_{3} v_{1}-r_{1} v_{3}\right) \hat{e}_{2} \\
& +\left(r_{1} v_{2}-r_{2} v_{1}\right) \hat{e}_{3}=\mathbf{r} \times \mathbf{v} .
\end{aligned}
$$

Por outro lado, o bivetor $\mathbf{r} \wedge \mathbf{v}$ e o vetor $\mathbf{r} \times \mathbf{v}$ possuem o mesmo módulo, conforme as equações (2) e (17). Assim o momento angular é o dual deste bivetor, isto é, $\mathscr{H}=$ $m[\star(\mathbf{r} \wedge \mathbf{v})]$.

\section{A Álgebra Geométrica de Clifford}

Em 1878 William Kingdon Clifford publicou um artigo no American Journal of Mathematics intitulado Applications of Grassmann's extensive algebra [34] apresentando uma nova estrutura matemática que ele denominou álgebra geométrica, mas que hoje também costuma ser conhecida como Álgebra de Clifford. O que Clifford fez foi sintetizar duas estruturas matemáticas aparentemente dissociadas: os quatérnions de Hamilton e a álgebra de extensão de Grassmann, mas por infelicidade o seu trabalho tampouco ganhou a devida atenção na época, sobretudo por causa de sua morte prematura que contribuiu para que seu trabalho caísse no esquecimento por algumas décadas.

Seu trabalho começou a ressurgir e ganhar maior importância a partir do final do século XX por ações do Prof. David Hestenes. Com esta visão mais abrangente da estrutura desta álgebra, verificou-se que a álgebra geométrica poderia ser usada como forma de unificar várias áreas da mecânica quântica e as aplicações nos mais variados temas e áreas contribuíram para uma grande e importante gama de trabalhos publicados recentemente [35 51]. Desde então, este assunto tem ganhado crescente importância. Além disto na descrição do momento angular, pode-se observar de forma clara, como a álgebra geométrica consegue em uma única estrutura unificar o seu conceito matemático e físico de forma clara, exata e concisa.

A álgebra de Clifford pode ser introduzida de forma mais geométrica [18, como o quociente da álgebra tensorial ou ainda a partir das álgebras de Grassmann [19]. O interesse no presente artigo é no produto geométrico e não 
será descrito detalhes das diversas formas de introduzir tal álgebra.

Supomos $V$ um espaço vetorial sobre $\mathbb{R}$ equipado com uma forma bilinear simétrica $g, \mathcal{A}$ uma álgebra associativa com unidade $\mathbf{1}_{\mathcal{A}}$ e ainda $\gamma$ uma aplicação linear $\gamma: V \rightarrow \mathcal{A}$. Uma álgebra de Clifford é o par $(\mathcal{A}, \gamma)$ para o espaço quadrático $(V, g)$ quando $\mathcal{A}$ é gerada como uma álgebra por $\{\gamma(\mathbf{u}) \mid \mathbf{u} \in V\}$ com $\gamma$ satisfazendo

$$
\gamma(\mathbf{u}) \gamma(\mathbf{w})+\gamma(\mathbf{w}) \gamma(\mathbf{u})=2 g(\mathbf{u}, \mathbf{w}) \mathbf{1}_{\mathcal{A}},
$$

para todo $\mathbf{u}, \mathbf{w} \in V$. Denotamos essa álgebra por $\mathcal{C} \ell(V, g)$.

O produto geométrico (ou Clifford) entre dois vetores $\mathbf{u}, \mathbf{w} \in V$ é dado por

$$
\gamma(\mathbf{u}) \gamma(\mathbf{w})=\gamma(g(\mathbf{u}, \mathbf{w}))+\gamma(\mathbf{u} \wedge \mathbf{w}) .
$$

O produto geométrico entre dois vetores pode ser visto como uma composição entre duas partes: a primeira é a imagem dentro de $\mathcal{C} \ell(V, g)$ da contração de $\mathbf{u}$ sobre w e a segunda parte é a imagem dentro de $\mathcal{C} \ell(V, g)$ do produto exterior.

Existe um isomorfismo de espaço vetorial entre a álgebra de Clifford e a álgebra de Grassmann e portanto o espaço de Clifford $\mathcal{C} \ell(V, g)$ possui uma estrutura multivetorial. Desta forma, os elementos da álgebra de Clifford são denominados também de multivetores. Nesta seção também será considerado um sistema euclidiano tridimensional, isto é $V=\mathbb{R}^{3}$, e uma base ortonormal representada por $\mathcal{B}=\left\{\hat{e}_{1}, \hat{e}_{2}, \hat{e}_{3}\right\}$, neste caso a álgebra de Clifford é denotada $\mathcal{C} \ell_{3}$. A relação 21) aplicada aos elementos dessa base pode ser escrita como

$$
\begin{aligned}
& \hat{e}_{i} \hat{e}_{i}=1 \quad \text { para } i=1,2,3 \\
& \hat{e}_{i} \hat{e}_{j}=-\hat{e}_{j} \hat{e}_{i} \text { para } i \neq j \text {. }
\end{aligned}
$$

O produto de Clifford, dado pela equação 22 , entre vetores pode ser reescrito como

$$
\mathbf{u w}=\mathbf{u} \cdot \mathbf{w}+\mathbf{u} \wedge \mathbf{w}
$$

Dentro da álgebra de Clifford também é possível definir a operação de dualidade e será denotado por *. Esta operação é análoga à chamada dualidade de Hodge dentro do cálculo com formas diferenciais 52. Dado um p-vetor $A_{p} \in \bigwedge\left(\mathbb{R}^{3}\right)$, com $p=0, \ldots, 3$, seu dual é definido como sendo

$$
\star A_{p}=(-1)^{\frac{p(p-1)}{2}} A_{p} \mathbb{I}
$$

onde II representa um trivetor simples unitário, isto é, $\mathbb{I}=\hat{e}_{1} \hat{e}_{2} \hat{e}_{3}$ denominado como pseudoescalar.

Um resultado importante obtido usando dualidade é, dados dois vetores $\mathbf{u}, \mathbf{w} \in \bigwedge\left(\mathbb{R}^{3}\right)$ pode-se relacionar o produto vetorial com o produto exterior

$$
\mathbf{u} \times \mathbf{w}=\star(\mathbf{u} \wedge \mathbf{w})=-(\mathbf{u} \wedge \mathbf{w}) \mathbb{I}
$$

sendo que a figura (7) mostra a interpretação geométrica desta relação.
Já a representação dos vetores posição $\mathbf{r}$ e velocidade $\mathbf{v}$ no espaço multivetorial $\bigwedge\left(\mathbb{R}^{3}\right)$ é da forma $\mathbf{r}=r_{1} \hat{e}_{1}+$ $r_{2} \hat{e}_{2}+r_{3} \hat{e}_{3}$ e $\mathbf{v}=v_{1} \hat{e}_{1}+v_{2} \hat{e}_{2}+v_{3} \hat{e}_{3}$.

Calculando os produtos escalar e exterior e substituindo na equação 24 obtém-se a expressão do produto geométrico

$$
\begin{aligned}
\mathbf{r v} & =\mathbf{r} \cdot \mathbf{v}+\mathbf{r} \wedge \mathbf{v} \\
\mathbf{r v} & =\left(r_{1} v_{1}+r_{2} v_{2}+r_{3} v_{3}\right) \\
& +\left(r_{1} v_{2}-r_{2} v_{1}\right) \hat{e}_{1} \wedge \hat{e}_{2}+\left(r_{1} v_{3}-r_{3} v_{1}\right) \hat{e}_{1} \wedge \hat{e}_{3} \\
& +\left(r_{2} v_{3}-r_{3} v_{2}\right) \hat{e}_{2} \wedge \hat{e}_{3}
\end{aligned}
$$

Observa-se que ao aplicar a equação 24 nos elementos da base, tem-se

$$
\hat{e}_{i} \hat{e}_{j}=\hat{e}_{i} \cdot \hat{e}_{j}+\hat{e}_{i} \wedge \hat{e}_{j},
$$

e da relação (1) que

$$
\hat{e}_{i} \hat{e}_{j}=\hat{e}_{i} \wedge \hat{e}_{j} .
$$

Logo a equação 26 pode ser reescrita da forma

$$
\begin{aligned}
\mathbf{r v} & =\left(r_{1} v_{1}+r_{2} v_{2}+r_{3} v_{3}\right) \\
& +\left(r_{1} v_{2}-r_{2} v_{1}\right) \hat{e}_{1} \hat{e}_{2}+\left(r_{1} v_{3}-r_{3} v_{1}\right) \hat{e}_{1} \hat{e}_{3} \\
& +\left(r_{2} v_{3}-r_{3} v_{2}\right) \hat{e}_{2} \hat{e}_{3}
\end{aligned}
$$

Pode-se ver claramente na equação 27 que aparece o bivetor dado pelo produto exterior escrito em termos do produto geométrico. Esse bivetor representa, como já foi visto na seção anterior, de maneira mais precisa o momento angular pois $\mathscr{H}$ está associada com área ao invés de comprimento e os bivetores possuem naturalmente esta característica. Outra forma de ver isto é calcular o vetor momento angular através da operação de dualidade (25), isto é,

$$
\mathbf{r} \times \mathbf{v}=-(\mathbf{r} \wedge \mathbf{v}) \mathbb{I}
$$

e uma característica importante desta operação é que seu resultado não muda o sinal perante uma inversão espacial qua é a mesma característica do produto vetorial. A beleza do produto geométrico é que contém todas as informações entre os vetores $\mathbf{r}$ e $\mathbf{v}$ : a relação métrica e a relação vetorial.

\section{Conclusão}

Heaviside usou o eletromagnetismo como incentivo para elaborar seu produto vetorial na descrição das equações

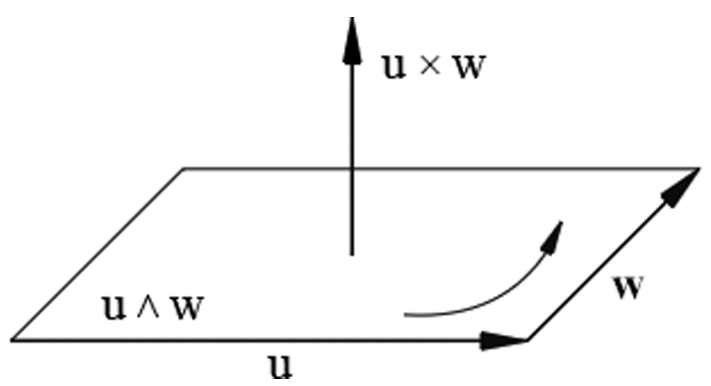

Figura 7: Representação geométrica do isomorfismo de Hodge. 
de Maxwell, obtendo uma praticidade operacional no tratamento destas equações. Porém, com as novas teorias físicas deve-se descrever com maior exatidão tais grandezas. Neste cenário, vale observar que o momento angular é uma grandeza que não atua ao longo de uma reta, mas sim normal a um plano. Logo da forma que é definido via álgebra vetorial, não é a melhor maneira de representá-lo. Na álgebra de Hamilton a parte vetorial da equação (10) representa, tanto algebricamente, quanto geometricamente, o momento angular descrito na álgebra vetorial. Porém, matematicamente, também não é a forma mais efetiva de representá-lo conforme mostramos neste artigo. Já na álgebra de Grassmann o produto entre dois vetores tem como resultado um bivetor, dado pela equação (15), que descreve naturalmente uma área orientada e possui a mesma estrutura , bastando aplicar o isomorfismo de Hodge para chegar a isto, além de possuir o mesmo módulo que a equação (7). Desta forma, um bivetor é a melhor maneira de representar matematicamente a grandeza física momento angular. Por outro lado, na álgebra geométrica de Clifford, o resultado do produto geométrico, além de ter o bivetor, que descreve a relação vetorial, possuí também um escalar, que descreve a relação métrica, unificando duas operações em um produto. Isto torna esta álgebra a mais completa para descrever a complexidade de diversas grandezas físicas importantes não só para físicos, como também engenheiros. Esta álgebra é mais geral e engloba todas as outras. Os autores em [19 mostram diversas representações da álgebra dos números complexos, $\mathcal{C} \ell_{0,1}$, da álgebra dos quatérnions, $\mathcal{C} \ell_{0,2}$ e uma construção da álgebra de Grassmann a partir do produto geométrico, uma vez que ambos possuem a mesma estrutura multivetorial. Vale ressaltar também que a álgebra vetorial só vale para espaços de dimensão três, enquanto a álgebra de Hamilton é válida para espaços de dimensão até quatro, por outro lado, as álgebras de Grassmann e Clifford são válidas para espaços de qualquer dimensão.

\section{Agradecimentos}

Os autores gostariam de agradecer ao apoio financeiro do CNPq dos Processos 404463/2016-9 e 307520/20161. O primeiro autor também gostaria de agradecer ao Departamento de Matemática da Universidade Federal do Mato Grosso, Campus de Rondonópolis, por ter concedido afastamento para o curso de pós-graduação na UNESP - Ilha Solteira. Os autores também agradecem os comentários e sugestões pertinentes do árbitro da Revista Brasileira de Ensino de Física e do Editor.

\section{Referências}

[1] J. Lekner, Journal of Physics: Condensed Matter 18, 6149 (2006).

[2] R. Wunenburger, J.I.V. Lozano e E. Brasselet, New Journal of Physics 17, 103022 (2015).
[3] D. Singh e N. Mobed, Classical and Quantum Gravity 28, 105024 (2011).

[4] L. Marrucci, E. Karimi, S. Slussarenko, B. Piccirillo, E. Santamato, E. Nagali e F. Sciarrino, Journal of Optics 13, 64001 (2011).

[5] V. Garcés-Chávez, D. McGloin, M.D. Summers, A. Fernandez-Nieves, G.C. Spalding, G. Cristobal e K. Dholakia, Journal of Optics A: Pure and Applied Optics 6, S235 (2004).

[6] J.P. Silva e J.M. Tavares, American Journal of Physics 75, 53 (2007).

[7] S.J. Robinson, Physics Education 49, 144 (2014).

[8] T.J. Hendrickson, American Journal of Physics 33, iii (1965).

[9] S. Datta, American Journal of Physics 46, 1190 (1978).

[10] J. Lindén, Physics Education 53, 23004 (2017).

[11] R.M. Sutton, American Journal of Physics 26, 580 (1958).

[12] R.B. Prigo e M. Reading, American Journal of Physics 45, 636 (1977).

[13] T.M. Kalotas e A.R. Lee, American Journal of Physics 58, 80 (1990).

[14] L.J. Pellizza, M.G. Mayochi, L.C. Brazzano e S.E. Pedrosa, American Journal of Physics 83, 1019 (2015).

[15] G.S. Krishnaswami e S. Sachdev, Resonance 21, 529 (2016).

[16] J. Vaz Jr., Revista Brasileira de Ensino de Física 22, 5 (2000).

[17] F. Brackx, R. Delanghe e F. Sommen, Pitman Books Limited 76, 308 (1982).

[18] J. Vaz Jr., Revista Brasileira de Ensino de Física 19, 234 (1997).

[19] J. Vaz e R. Rocha Jr., Algebras de Clifford e Espinores (Editora Livraria da Física, São Paulo, 2012).

[20] M.J. Crowe, A history of vector analysis: The evolution of the idea of a vectorial system (Notre Dame University Press, London, 1967).

[21] J.W. Gibbs, Elements of vector analysis: arranged for the use of students in physics (Tuttle, Morehouse \& Taylor, New Haven, 1884).

[22] J.W. Gibbs, The scientific papers of Josiah Willard Gibbs (Dover, New York, 1961).

[23] O. Heaviside, Electrical Papers (Chelsea Publishing Company, New York, 1970).

[24] S. Verma, Ideias Geniais. Os principais teoremas, teorias, leis e princípios científicos de todos os tempos (Editora Gutenberg, Belo Horizonte, 2011).

[25] I.D. Camargo e P. Boulos, Geometria analítica: um tratamento vetorial (Editora Pearson Education, São Paulo, 2005).

[26] P. Winterle, Vetores e geometria analítica (Editora Pearson Makron Books, São Paulo, 2014).

[27] J. Walker, Fundamentos de Física (Editora LTC, Rio de Janeiro, 2016).

[28] H.M. Nussenzveig, Curso de Física Básica: Mecânica (Editora Edgard Blucher, São Paulo, 2013).

[29] W.R. Hamilton, Elements of quaternions (Chelsea Publishing Company, New York, 1969).

[30] J. Kuipers, Quaternions and Rotation Sequences: A Primer with Applications to Orbits, Aerospace, and Virtual Reality (Princeton University Press, Princeton, 1999). 
[31] W.R. Hamilton, in Proceedings of the Royal Irish Academy, Dublin, 1847 (Royal Irish Academy, Dublin, 1847).

[32] H.G. Grassmann, Die Lineale Ausdehnungslehre (Otto Wigand, Leipzig, 1844).

[33] E.L. Lima, Álgebra Exterior (IMPA, Rio de Janeiro, 2009).

[34] W.K. Clifford, American Journal of Mathematics 1, 350 (1878).

[35] D. Hestenes e A.N. Lasenby, Space-time algebra, (Gordon and Breach, New York, 1966).

[36] D. Hestenes, Journal of Mathematical Physics 8, 798 (1967).

[37] D. Hestenes, Journal of Mathematical Physics 15, 1768 (1974).

[38] D. Hestenes, Foundations of Physics 12, 153 (1982).

[39] D. Hestenes e G. Sobczyk, Clifford algebra to geometric calculus: a unified language for mathematics and physics (D. Reidel Publishing Company, Dordrecht, 1984).

[40] D. Hestenes, Foundations of Physics 15, 63 (1985).

[41] D. Hestenes, in: Clifford Algebras and their Applications in Mathematical Physics, editado por J.S.R. Chisholm e A.K. Common (Springer, Dordrecht, 1986), v. 183, p. 321.

[42] D. Hestenes, New foundations for classical mechanics (Kluwer Academic Publishers, Dordrecht, 1986).

[43] D. Hestenes, in: Clifford Algebras and their Applications in Mathematical Physics, editado por F. Brackx, R. Delanghe e H. Serras (Springer, Dordrecht, 1993), v. 55, p. 269.

[44] D. Hestenes, Advances in Applied Clifford Algebras 7, 97, (1997).

[45] D. Hestenes, in: Clifford Algebras and their Applications in Mathematical Physics, editado por V. Dietrich, K. Habetha e G. Jank (Springer, Dordrecht, 1998), v. 94, p. 129.

[46] D. Hestenes, in: Geometric Algebra with Applications in Science and Engineering, editado por E.B. Corrochano e G. Sobczyk, (Birkhäuser, Boston, 2001), p. 3.

[47] D. Hestenes, Foundations of Physics 35, 903 (2005).

[48] D. Hestenes e J.W. Holt, Journal of Mathematical Physics 48, 23514 (2007).

[49] D. Hestenes, in: Geometric Algebra Computing, editado por E. Bayro-Corrochano e G. Scheuermann (Springer, London, 2010), p.3.

[50] D. Hestenes, Advances in Applied Clifford Algebras 24, 257 (2014).

[51] D. Hestenes, Advances in Applied Clifford Algebras 27, 351, (2017).

[52] H. Flanders, Differential Forms with Applications to the Physical Sciences (Academic Press, New York, 1963). 